\title{
New in vitro system to predict chemotherapeutic efficacy of drug combinations in fresh tumor samples
}

\author{
Frank Christian Kischkel ${ }^{\text {Corresp., }}{ }^{1}$ ， Julia Eich ${ }^{1}$, Carina I Meyer ${ }^{1}$ ， Paula Weidemüller ${ }^{1}$, Jens Krapfl ${ }^{1}$, Rauaa \\ Yassin-Kelepir ${ }^{1}$, Laura Job ${ }^{1}$, Marius Fraefel ${ }^{1}$, Ioanna Braicu ${ }^{2}$, Annette Kopp-Schneider ${ }^{3}$, Jalid Sehouli ${ }^{2}$, \\ Rudy Leon De Wilde ${ }^{4}$ \\ 1 TherapySelect, Heidelberg, Germany \\ 2 Gynecology Department, Charité Berlin, Virchow Campus Berlin, Germany \\ 3 Division of Biostatistics, German Cancer Research Center, Heidelberg, Germany \\ 4 University Hospital for Gynecology, Carl von Ossietzky University Oldenburg, Germany \\ Corresponding Author: Frank Christian Kischkel \\ Email address: frank.kischkel@therapyselect.de
}

Background. To find the best individual chemotherapy for cancer patients, the efficacy of different chemotherapeutic drugs can be predicted by pretesting tumor samples in vitro via the chemotherapyresistance (CTR)-Test ${ }^{\circledast}$. Although drug combinations are widely used among cancer therapy, so far only single drugs are tested by this and other tests. However, several first line chemotherapies are combining two or more chemotherapeutics, leading to the necessity of drug combination testing methods.

Methods. We established a system to measure and predict the efficacy of chemotherapeutic drug combinations with the help of the Loewe additivity concept in combination with the CTR-test. A combination is measured by using half of the monotherapy's concentration of both drugs simultaneously. With this method the efficacy of a combination can also be calculated based on single drug measurements.

Results. The established system was tested on a data set of ovarian carcinoma samples using the combination carboplatin and paclitaxel and confirmed by using other tumor species and chemotherapeutics. Comparing the measured and the calculated values of the combination testings revealed a high correlation. Additionally, in $70 \%$ of the cases the measured and the calculated values lead to the same chemotherapeutic resistance category of the tumor.

Conclusion. Our data suggest that the best drug combination consists of the most efficient single drugs and the worst drug combination of the least efficient single drugs. Our results showed that single measurements are sufficient to predict combinations in specific cases but there are exceptions in which it is necessary to measure combinations, which is possible with the presented system. 
1 New In Vitro System to Predict Chemotherapeutic

2 Efficacy of Drug Combinations in Fresh Tumor

3 Samples

\section{Author Names and Affiliations}

5 Frank Christian Kischkel ${ }^{1)}$, Julia Eich ${ }^{1)}$, Carina Meyer ${ }^{1)}$, Paula Weidemüller ${ }^{1)}$, Jens

$6 \mathrm{Krapf}^{1}$, Rauaa Yassin-Kelepir ${ }^{1)}$, Laura $\mathrm{Job}^{1)}$, Marius Fraefel ${ }^{1)}$, loanna Braicu ${ }^{2)}$, Annette

7 Kopp-Schneider ${ }^{3)}$, Jalid Sehouli'², Rudy Leon De Wilde ${ }^{4)}$

8 1) TherapySelect, Heidelberg, Germany

9 2) Charité Berlin, Gynecology Department, Virchow Campus Berlin, Germany

10 3) German Cancer Research Center, Division of Biostatistics, Heidelberg, Germany

11 4) University Hospital for Gynecology, Carl von Ossietzky University Oldenburg,

12 Germany

14 Background. To find the best individual chemotherapy for cancer patients, the efficacy 
15 of different chemotherapeutic drugs can be predicted by pretesting tumor samples in

16 vitro via the chemotherapy-resistance (CTR)-Test ${ }^{\circledR}$. Although drug combinations are

17 widely used among cancer therapy, so far only single drugs are tested by this and other

18 tests. However, several first line chemotherapies are combining two or more

19 chemotherapeutics, leading to the necessity of drug combination testing methods.

20 Methods. We established a system to measure and predict the efficacy of

21 chemotherapeutic drug combinations with the help of the Loewe additivity concept in

22 combination with the CTR-test. A combination is measured by using half of the

23 monotherapy's concentration of both drugs simultaneously. With this method the efficacy

24 of a combination can also be calculated based on single drug measurements.

25 Results. The established system was tested on a data set of ovarian carcinoma

26 samples using the combination carboplatin and paclitaxel and confirmed by using other

27 tumor species and chemotherapeutics. Comparing the measured and the calculated

28 values of the combination testings revealed a high correlation. Additionally, in $70 \%$ of

29 the cases the measured and the calculated values lead to the same chemotherapeutic

30 resistance category of the tumor.

31 Conclusion. Our data suggest that the best drug combination consists of the most

32 efficient single drugs and the worst drug combination of the least efficient single drugs.

33 Our results showed that single measurements are sufficient to predict combinations in

34 specific cases but there are exceptions in which it is necessary to measure

35 combinations, which is possible with the presented system. 


\section{Introduction}

37 Progressively more is known about the underlying causes for the formation of a tumor,

38 which are highly complex and involve in general several mechanisms at the molecular

39 level. Therefore, tumors are heterogeneous and every individual patient has a specific

response profile to the selected treatment concerning the varying chemosensitivity of

41 tumor cells (Blom et al., 2016). However, the standard therapy for cancer treatment is

42 based on an average response of a group of patients with similar tumor types which

43 leads to a lack of benefit in several cases and to the necessity of a next-line therapy

44 (Blom et al., 2016). One method to improve the therapeutic outcome is to priory assess

45 the resistance of tumors from individual patients to chemotherapeutic drugs in order to

46 provide the most efficient therapy to every single patient (Nygren \& Larsson, 2008).

47 Such therapy selection strategies are considered to have great potential to advance

48 cancer treatment (Pusztai et al., 2004; Ludwig \& Weinstein, 2005; loannidis, 2007;

49 Trusheim, Berndt \& Douglas, 2007). A recent prospective clinical trial showed the benefit

50 of using an in vitro chemotherapeutic test in ovarian cancer (Rutherford et al., 2013;

51 Grendys et al., 2014). This relationship can be intuitively explained by the assumption

52 that if a drug is ineffective in a simple system like an in vitro test using isolated tumor

53 cells, the probability that is has an effect in a patient is highly unlikely (Nygren et al.,

54 1994). One option of such an in vitro test is the so-called Chemotherapy Resistance Test

55 (CTR-Test $\left.^{\circledR}\right)$ which was the chosen method in this paper. The CTR-Test is identical to a

56 formerly described extreme drug resistance (EDR) assay (Kern \& Weisenthal, 1990).

57 EDR assays are applied to identify chemotherapeutics which are ineffective rather than

58 to find chemotherapeutics which are likely to show an effect. Thereby, the treatment of a

59 patient with a toxic agent that does not result in a therapeutic benefit can be prevented 
60 (Tattersall \& Harnett, 1986; Myers et al., 1987; Beck, 1987). It is known that the

61 capability to predict drug resistance is presumably $>95 \%$ whereas the ability to predict

62 chemo-sensitivity lies around $60 \%$ (Kim et al., 2009). The CTR-Test shows a >99\%

63 accuracy in finding ineffective chemotherapeutics that do not produce a clinical response

64 (Kern \& Weisenthal, 1990).

65 So far only single drugs are tested in this system. However, in the case of ovarian

66 cancer the standard first-line treatment is a combination therapy of carboplatin together

67 with paclitaxel (du Bois et al., 2003; Pfisterer et al., 2006; du Bois et al., 2006; Bookman

68 et al., 2009). In clinical practice, combination therapies are more frequently applied and

69 in general the benefits of a combination therapy are reduced side effects as well as

70 reduced drug resistance. The reason for reduced side effects is that lower doses of the

71 two drugs can be applied which still lead to the same efficacy as a higher dose of the

72 particular monotherapy but avoids toxicity. Reduced drug resistance is achieved by

73 diverse mechanisms of action of the two chemotherapeutics (Sparano, 1999; Prisant,

74 2002; Tallarida, 2006; Kashif et al., 2015).

75 There are several paper published showing that it is sufficient to test single drugs via the

76 CTR-Test and use their efficacy data to find effective combination therapies, which lead

77 to a clinical response (Mehta et al., 2001; Holloway et al., 2002; Loizzi et al., 2003;

78 d'Amato et al., 2009; Kim et al., 2009; Matsuo et al., 2009). The question arises whether

79 in general there would be an improvement in cancer therapy when combinations instead

80 of single drugs are tested or if the testing of single drugs is sufficient for a good clinical

81 prediction. To our knowledge no effective in vitro test system or test principle for testing

82 drug combinations exists. Therefore, there is need of an enhanced in vitro diagnostic

83 test system which enables the clinically relevant investigation of the efficacy of drug

84 combinations. In this paper we used a new system to test drug combinations in vitro with 
85 the CTR-Test.

\section{Material and Methods}

87 Tumor Tissue Samples Collection

88 The tumor specimen were collected as part of the commercially offered CTR-Test or as

89 part of a clinical trial. All included samples were left over after the commercial or clinical

90 trial assay was performed. For all samples patient's consent forms exist, which allow

91 further scientific investigations. In total 273 ovarian carcinoma, 1 malignant melanoma, 1

92 small cell lung cancer, 1 mamma carcinoma, 4 colon carcinoma and 1 NSCLC biopsies

93 were collected. After surgery tissue samples were directly stored in medium and sent to

94 the laboratory (TherapySelect, Heidelberg, Germany) to perform the CTR-Test. The

95 specimens arrived at TherapySelect within $24 \mathrm{~h}$ and were processed on the same day.

\section{CTR-Test}

97 The tissues were processed and the CTR-Test was performed by TherapySelect,

98 according to a published protocol (Kern \& Weisenthal, 1990; d'Amato et al., 2009).

99 Briefly, fresh tumor material is minced into single cells and small cellular aggregates

100 (spheroids). Viability and percentage of tumor cells is determined by an external

101 pathology. Cells are seeded in a culture dish, in which they cannot adhere, and directly

102 treated with a specific chemotherapeutic drug or a drug combination. After $72 \mathrm{~h}$

103 incubation tritiated thymidine $\left(\mathrm{H}^{3}\right.$-Thymidine) is added to the cells. After additional $48 \mathrm{~h}$ 
104 cells are harvested onto glass fiber filters and the isotope uptake into the DNA is

105 analyzed by scintillation counting. The data obtained are counts per minute (cpm). Cells

106 cultured without drugs are used as negative control and cells treated with a lethal dose

107 are the positive control. The chemotherapeutic effect is measured in percent cell growth

108 inhibition $(\mathrm{PCl})$ using the formula: $\mathrm{PCl}=(\mathrm{cpm}($ treated cells $)-c p m($ positive control $))$

109 (cpm(negative control)-cpm(positive control).

110 Drugs Used For Analysis

111 All drugs used in this study were selected for therapeutic relevance and were validated

112 for the CTR-Test before the analysis of the tumor samples for this study. For the

113 validation, various drug concentrations were tested in the CTR-Test with freshly isolated

114 tumor samples in order to find a concentration which shows a sufficient distribution of

115 drug action among the tumor samples. Final applied drug concentrations are presented

116 in Table 1. For the measurements of drug combinations (two drugs) half of the

117 concentration of each single drug was used to treat the cells simultaneously.

\section{Statistical Analysis}

$119 \mathrm{PCl}$ values were obtained on the response rate of a tumor sample collective to a certain

120 concentration of a chemotherapeutic drug. Frequency distributions of $\mathrm{PCl}$ values were

121 generated by joining the mid-points of 4- or 5-bin histograms by a smooth curve in

122 Excel.

123 The frequency distributions were applied to identify the three resistance categories SR,

$124 \mathrm{MR}$ and ER. Therefore, the mean $(\mu)$ and the standard deviation (SD) were determined.

125 Mean values are presented in the corresponding figures, SD values can be found in

126 Supplemental Table 1. ER is characterized as $\mathrm{PCl}<\mu-1 \mathrm{SD}, \mathrm{MR}$ as $\mathrm{PCl}>\mathrm{ER}$ but $<\mu$ 
127 and $\mathrm{SR}$ as $\mathrm{PCl} \geq \mu$.

128 We compared the measured and calculated $\mathrm{PCl}$ values of the different combinations by

129 determining the Pearson correlation coefficient. To assess agreement between

130 calculated and measured values we showed the difference of calculated and measured

131 values vs. the average of both values in a Bland-Altman Plot (Supplemental Figure 1).

\section{Human Studies}

133 TherapySelect offers the commercial testing of drug efficacies for viable tumor samples.

134 For this testing viable tumor specimen is shipped to TherapySelect's laboratory.

135 Customers (patients) fill out a consents and order form. In this form there is a section in

136 which the patients can choose whether left over material can be used for research

137 purposes. For all used samples patient's consent forms exist, which allow further

138 scientific investigations. For this paper no Ethical approval was requested, since human

139 tissue was initially removed for commercially performed diagnostic purposes.

\section{$140 \quad$ Results}

141 New System to Measure and Calculate Efficacy of Drug Combinations and

142 Determination of the Correlation

143 The response rate of a collective of tumor samples to a certain concentration of a

144 chemotherapeutic drug experiences a standard distribution. An ideal testing

145 concentration of a drug is found if the histogram spans over the full spectrum of percent 
146 cell growth inhibition (PCI) and the curve's mean overlaps with $50 \%$ inhibition effect.

147 Figure 1A shows an ideal distribution with the perfect concentration. This concept is 148 used to find and validate concentrations for single drug therapy testing. The chosen

149 concentration (Table 1) is in a physiological range, that means close to or below the

150 maximal serum concentration for the individual drugs.

151 In case of combination testing the individual concentrations have to be adapted.

152 Therefore, the concept of Loewe additivity was customized to this question. The 153 underlying assumption of the additivity model is that two inhibitors operate through

154 similar mechanisms on a target and dose substitution is the following consequence 155 (Loewe, 1953; Berenbaum, 1989). Adapting this concept to our issue, in an ideal case 156 the distribution curves of separately measured drugs, A and B, used at the ideal 157 concentration reveal two identical response histograms. Therefore, one half of the ideal 158 concentration of drug A could replace one half of the ideal concentration of drug B or 159 vice versa. This would lead to a histogram identical to a curve produced by the full 160 concentration of drug A or B, respectively.

161 With this in mind the extent of inhibition in combination $(\mathrm{PCl}(\mathrm{a}+\mathrm{b}))$ can be calculated by 162 a mathematical model based on the inhibition effects (PCla or PClb) of the mono-drugs 163 (A or B) (Figure 1A)

$$
\mathrm{PCl}(\mathrm{a}+\mathrm{b})=\frac{P C I a}{2}+\frac{P C I b}{2}
$$

165 The inhibition effects of the two mono-drugs (PCla and PClb) are divided by two 166 because half of the concentration of drug A and drug B would be used if they would be 167 applied in combination. Both values are added and result in the inhibition effect of the 168 combination $(\mathrm{PCl}(\mathrm{a}+\mathrm{b}))$. 
169 In an ideal setting the value of the measured combination effect is equal to the

170 calculated effect. In an ideal plot of measured versus calculated effect, the correlation

171 coefficient would be 1 and is linear correlated, which represents the additive effect

172 (Figure 1B). In this ideal setting a data point either above or below the line would stand

173 for an antagonistic or a synergistic effect, respectively. In the curve representation of the

174 patients collective those effects are seen by a shift to the left or to the right of the

175 histogram in case of antagonism or synergy, respectively (Figure 1A). Synergy can be

176 defined as a stronger cell growth inhibition effect measured than the calculated

177 combination effect. Antagonistic would mean a reduced effect in combination (Chou,

178 2010; Kashif et al., 2014).

179 To test this theory, 273 ovarian carcinoma samples (99 primary, 140 recurrent and 34

180 unknown ovarian carcinoma cases) were treated with carboplatin and paclitaxel alone or

181 in combination. For the combination measurement half of the concentration of each

182 tested mono-drug (carboplatin/paclitaxel) was applied. Cell growth inhibition effects were

183 measured with the CTR-Test and histograms were created. Additionally, the cell growth

184 inhibition effect was calculated for the combination by using the presented formula. The

185 four different distributions of effect in the tumor sample collective are presented in

186 Figure 1C. The curve of carboplatin is slightly shifted compared to the histogram of

187 paclitaxel. However, the calculated curve lies in between the single drug histograms and

188 the calculated curve is in general more narrow. As mentioned before, the chosen

189 chemotherapeutic concentration should lead to a mean at a $\mathrm{PCl}$ value of $50 \%$ in the

190 histogram of the collective. However all curves have a mean which spreads around 70

$191 \%$ (Figure 1C). The calculated mean $69.3 \%$ of the combination lays between the mean

192 of carboplatin and paclitaxel with $67.7 \%$ and $70.9 \%$, respectively. The measured value

193 lays above the calculated mean with $73.0 \%$. To further analyze the accuracy of 
194 predicting the combination effect, the calculated and measured PCI values are plotted

195 against each other. (Figure 1D). The combination of carboplatin and paclitaxel leads to

196 a correlation of 0.84 (Table 2).

197 Evaluation of Predicting Efficacy by Measured Drug Combinations versus the 198 Calculated on Basis of Single Drug Measurement

199 To classify the chemoresistance of tumors to certain drugs, the response histogram of a 200 patient collective is used to define three resistance categories (Mehta et al., 2001;

201 Holloway et al., 2002; Loizzi et al., 2003; Kim et al., 2009; Matsuo et al., 2009). Extreme 202 resistance $(\mathrm{ER})$ is marked by $\mathrm{PCl}<$ Mean $-1 \mathrm{SD}$ (standard deviation). $\mathrm{PCl}>\mathrm{ER}$ but $<$ 203 Mean values are classified as medium resistance (MR). All $\mathrm{PCl} \geq$ Mean values are

204 called slight resistance (SR) (Figure 2A). Mean values used for determination of 205 resistance categories are presented in the corresponding figures, the standard 206 deviations can be found in Supplemental Table 1. Those resistant classifications are 207 used to predict treatment success by the use of a monotherapy for the individual patient 208 (Kern \& Weisenthal, 1990).

209 The influence of the single drug resistance in the combination therapy was analyzed.

210 Therefore, the four histograms of carboplatin, paclitaxel, measured and calculated

211 values, were used to define the $\mathrm{PCl}$ values of the resistance borders. In the comparison

212 plot of measured versus calculated $\mathrm{PCl}$, lines are drawn at the borders between ER and

213 MR (red) and between MR and SR (green) for calculated and measured individually. As

214 an example how the resistance borders were defined, it is shown how the resistance

215 borders for the measured combination (carboplatin and paclitaxel) were determined. The

216 Mean value $(\mu)$ is $73.3 \%$ (see Figure 1) and indicates the border between SR and MR

217 (green line). The standard deviation (SD) is $14.9 \%$ (see Supplemental Table 1) and 
218 therefore the border between MR and ER ( $\mu-1$ SD) lies at $58.4 \%$ (red line). The three

219 resulting squares along the diagonal represent overlapping resistance profiles, gained

220 by the measured and the calculated resistance classification (Figure 2B). In this data

221 set in $77 \%$ of the cases the resistance classification of the measured resistance is equal

222 to the calculated resistance (Table 2).

223 Additionally, each data point was categorized by the underlying resistance classes of the

224 single drug measurements (Figure 2C). Comparing single drug resistance categories to

225 the measured combination-categories reveals that if carboplatin and paclitaxel are

226 identical categorized either as SR or ER, the measured resistance category stays in 95

$227 \%$ or $100 \%$ of the cases SR or ER, respectively. A similar tendency is seen in the case

228 of medium resistance for carboplatin and paclitaxel. Here $75 \%$ of the measured

229 samples are categorized as MR. However, for combinations of different single resistance

230 categories the prediction of the resistance profile for the measured combination is less

231 accurate. For the far apart combinations ER with SR there is almost no prediction

232 possible. For combinations closer together like ER with MR and SR with MR a tendency

233 can be seen (Figure 2C).

\section{Applying New System to other Drug Combinations}

235 The first line standard therapy for treating ovarian carcinoma is a combination therapy of 236 carboplatin together with paclitaxel. We could show that our new approach to use the

237 CTR-Test system for testing drug combinations is functional in this scenario. Most of the

238 measured combination $\mathrm{PCl}$ values are in agreement with the calculated ones, basing on

239 the single measurements. As mentioned above, for the relevant resistance categories

240 SR and ER almost all measured values coincide with the calculated values and belong

241 to the correct resistance category. Besides the combination carboplatin and paclitaxel 
242 also other chemotherapeutics can be applied to treat ovarian carcinoma. Therefore, we

243 tested carboplatin together with six other chemotherapeutics in the CTR-Test in order to

244 investigate if the new system is also functional in testing other carboplatin combinations.

245 The six chemotherapeutics were: Caelyx® (doxorubicin-hydrochloride in a pegylated

246 liposomal formulation), doxorubicin, docetaxel, etoposide, gemcitabine and topotecan.

247 Cell growth inhibition was determined by the CTR-Test. Due to tumor material limitations

248 only a subset of the 273 ovarian tumor samples was tested with the six carboplatin

249 combinations. 39 ovarian tumor samples were measured for the combination

250 carboplatin-Caelyx (Figure 3A and E), 29 for carboplatin-doxorubicin (Figure 3B and

251 F), 30 for carboplatin-docetaxel (Figure 4A and C), 32 for carboplatin-etoposide (Figure

252 3C and G), 36 for carboplatin-gemcitabine (Figure 4B and D) and 29 for carboplatin-

253 topotecan (Figure 3D and H). Histograms for the single drugs and in combination were

254 determined and the combined $\mathrm{PCl}$ value was calculated using the formula presented

255 above. The histograms of the combinations carboplatin-Caelyx, carboplatin-doxorubicin,

256 carboplatin-etoposide and carboplatin-topotecan show a good distribution of the different

$257 \mathrm{PCl}$ curves for the single drugs, the calculated and the measured values (Figure 3A-D).

258 However, the histograms for the combinations carboplatin-docetaxel and carboplatin-

259 gemcitabine exhibit a distribution which is divergent in a great extent from an ideal

260 distribution (Figure 4A and B).

261 The measured and calculated PCl values were plotted against each other to investigate

262 how precise the calculated $\mathrm{PCl}$ of the combination was determined. In accordance with

263 the histograms, the data points for the combinations carboplatin-Caelyx, carboplatin-

264 doxurubicin, carboplatin-etoposide and carboplatin-topotecan spread around a

265 regression line and show a similar distribution as the combination carboplatin-paclitaxel

266 (Figure 3E-H). The data points for the combinations carboplatin-docetaxel and 
267 carboplatin-gemcitabine show a different pattern (Figure 4C and D). In Table 2 the

268 correlation coefficients $\mathrm{R}$ as well as the accuracy of resistance classification of the

269 calculated versus the measured values is illustrated.

270 In order to investigate if our test system is also functional in a carboplatin-independent

271 system, we tested two other drug combinations, 5-fluorouracil - SN-38 (active form of

272 the prodrug irinotecan) (Figure 5A and $\mathbf{C}$ ) and 5-fluorouracil - oxaliplatin (Figure 5B

273 and D). These two combinations are standard therapies for colon carcinoma. Therefore,

274 additional to the ovarian carcinomas other tumor types were measured as well. For the

275 combination 5-fluorouracil - SN-38 32 ovarian carcinoma, 1 melanoma, 1 small cell

276 bronchial carcinoma, 1, non-small cell lung carcinoma, 1 mamma carcinoma and 4 colon

277 carcinoma were used. For the combination 5-fluorouracil - oxaliplatin 31 ovarian

278 carcinoma, 1 melanoma, 1 small cell bronchial carcinoma, 1 non-small cell lung

279 carcinoma, 1 mamma carcinoma and 2 colon carcinoma were tested. Cell growth

280 inhibition was determined by the CTR-Test. Due to the fact that from colon carcinoma

281 samples only a limited number of cells can be isolated and the samples were needed for

282 regular commercial testing we used only 6 left over colon carcinoma samples for testing

283 the aforementioned two combinations. We also used ovarian carcinoma samples and

284 other tumor types, which are left overs of commercial CTR-Tests performed in the lab.

285 Data analysis was performed like for the carboplatin combinations with regard to 286 frequency distributions and the correlation between calculated and measured $\mathrm{PCl}$

287 values (Figure 5). The histograms of both combinations show a distribution of the 288 different $\mathrm{PCl}$ curves of the single drugs, the calculated and the measured values, which

289 differs from an ideal distribution (Figure 5A and B). The data points for both the 290 combination 5-fluorouracil - SN-38 and 5-fluorouracil - oxaliplatin show a good 291 distribution in the SR range. However, the rest of the data points show a divergent 
292 pattern (Figure 5C and D). Though, the correlation coefficient and the resistance

293 classification between calculated and measured values (Table 3) exhibit values in a

294 good range.

295 To adequately compare calculated and measured $\mathrm{PCl}$ values, we generated Bland-

296 Altman-Plots for the different combinations (Supplemental Figure 1). For the

297 combinations carboplatin and paclitaxel (Supplemental Figure 1A), carboplatin and

298 Caelyx (Supplemental Figure 1B), carboplatin and etoposide (Supplemental Figure

299 1D), carboplatin and topotecan (Supplemental Figure 1E) as well as 5-fluorouracil and

$300 \mathrm{SN}-38$ (Supplemental Figure $1 \mathrm{H}$ ) at $3 \%$ to $7 \%$ on the average, the calculated values

301 are higher than the measured values. Furthermore, $95 \%$ of the deviations between

302 measured and calculated values lie between $+27 \%$ and $-14 \%$, except for 5 -fluorouracil

303 and SN-38 where the values lie between $+31 \%$ and $-26 \%$.

304 The combination carboplatin and doxorubicin (Supplemental Figure 1C) shows a very

305 good agreement between measured and calculated values. At only $0,7 \%$ on the

306 average, the calculated values are higher than the measured values. $95 \%$ of the

307 deviations between measured and calculated values lie between $+16 \%$ and $-15 \%$.

308 In agreement with our other results, the combinations carboplatin and docetaxel

309 (Supplemental Figure 1 F) and carboplatin and gemcitabine (Supplemental Figure

310 1G) exhibit worse values. At $12 \%$ and $11 \%$ on the average, the calculated values are

311 higher than the measured values. $95 \%$ of the deviations between measured and

312 calculated values lie between $+35 \%$ and $-10 \%$ or $+33 \%$ and $-11 \%$, respectively.

313 For the combination 5-fluorouracil and oxaliplatin (Supplemental Figure 1I), at $3 \%$ on

314 the average, the calculated values are lower than the measured values. This is in

315 contrast to the other combinations where the calculated values are mostly higher than

316 the measured ones. $95 \%$ of the deviations between measured and calculated values lie 
317 between $+48 \%$ and $-53 \%$.

\section{Discussion}

319 We developed a new system which allows to test the clinical relevance of drug 320 combinations in vitro via the CTR-test and a formula based on an additive model. Our

321 system uses tumor material from patients instead of cell lines and the drugs are applied

322 in concentrations that are similar to or lower than the physiological maximal serum

323 concentrations $\left(C_{\max }\right)$ which leads to a high comparability to clinical data. Additionally,

324 this system uses a quite simple mathematical model which is based on the concept of

325 Loewe additivity (Loewe, 1953; Berenbaum, 1989) Our adapted concept states that one

326 half of the ideal concentration of drug A could replace one half of the ideal concentration

327 of drug B or vice versa. The adapted concept is employed to predict the efficacy of a

328 combination by calculating its $\mathrm{PCl}$ value based on single drug measurements. In an

329 additive situation one half of the concentration of each drug applied in combination leads

330 to an equal PCl of the drugs alone at full concentration (Fig. 1 A and B).

331 To test our system we used a set of 273 ovarian carcinoma samples and measured the

332 resistance profiles of carboplatin and paclitaxel alone or in combination by the CTR-Test.

333 The adapted concept was used to calculate both the combination concentration and its

$334 \mathrm{PCl}$ value. The setting of ovarian carcinoma was chosen because it is standardly treated

335 with carboplatin and paclitaxel in combination. The aim of this test was to verify the

336 accuracy of calculated PCls of the combination based on the single drug

337 measurements. Therefore, calculated and measured $\mathrm{PCl}$ values of the combination 
338 were compared (Figure 1D). Since the data exhibit a correlation coefficient of 0.84

339 (Table 2) our system shows to be highly functional in predicting the combination PCI

340 values based on the single drug measurements in the case of carboplatin and paclitaxel.

341 This high correlation coefficient indicates a close relationship between the conceived

342 ideal case and the actual activity of the two drugs combined. This is proven by the

343 similarity between the frequency distributions of the $\mathrm{PCl}$ values and the theoretical ideal

344 distributions (Figure 1C). Consequently, the combination of carboplatin and paclitaxel

345 functions in an additive way and the corresponding $\mathrm{PCl}$ value can be calculated.

346 An important step for predicting the efficacy of a chemotherapy is the resistance

347 classification of tested drugs and their combinations based on $\mathrm{PCl}$ values. In detail, the

348 resistance categories are determined individually via the frequency distribution of

349 carboplatin, paclitaxel and the measured and calculated combination (Figure 2A). Due

350 to the importance of a correct classification the conformity of the measured and the

351 calculated based classification was tested. The calculated resistance classification of the

352 combination carboplatin and paclitaxel is in almost $80 \%$ of the cases in agreement with

353 the actually measured resistance category (Figure 2B and Table 2). This leads to the

354 assumption that it is feasible and sufficient to predict the chemoresistance of a tumor to

355 a drug combination by measuring the single drugs. To test this assumption the

356 measured resistance category was compared with the underlying single drug resistance

357 classification. The postulated assumption holds true when the two individual drugs both

358 belong to the same resistance category in case of SR and ER. However, when the

359 resistance categories differ or both are MR, the prediction of the resistance category of

360 the combination is less precise (Figure 2C).

361 This suggests that if a patient is to be treated with a combination of drugs and the single 362 drug measurements result in the same resistance categories (SR, ER), the calculated 
363 resistance category most likely leads to a clinical benefit or to no clinical benefit of the

364 patient, respectively. If both drugs are rated SR or ER, the patient can be treated with

365 the corresponding combination or should get another combination, respectively. Taken

366 together, our data suggest that the best combination is composed of the most efficient

367 and the worst combination is composed of the least efficient single drugs. For MR cases

368 it should be explored if there may be a more functional combination. In case of different

369 resistance categories of the single drugs it makes sense to also test the combination

370 because there is no precise prediction possible about the efficacy of the combination.

371 Those conclusions are so far only based on the combination of carboplatin and

372 paclitaxel. In order to prove our system in a wider range we tested other carboplatin

373 combinations applied in ovarian cancer treatment (Figure 3). Looking at the correlation

374 coefficients of the combinations carboplatin and Caelyx, carboplatin and doxorubicin,

375 carboplatin and etoposide as well as carboplatin and topotecan, confirms the correlation

376 seen for the carboplatin - paclitaxel combination (Table 2). Furthermore, the frequency

377 distributions show an almost ideal distribution (Figure 3A - D) and the calculated

378 resistance classifications are in around $70 \%$ of the cases equal to the measured

379 classifications (Table 2). Therefore, all previous assumptions could be confirmed by

380 testing other carboplatin combinations.

381 Moreover, it was verified if our system is also functional in a carboplatin independent 382 setting and for other tumor species. Thus, we examined the combinations of 5-

383 fluorouracil with SN-38 and of 5-fluorouracil with oxaliplatin, which are standard

384 therapies for colon carcinoma (Figure 5). The data points for the combinations 5-

385 fluorouracil and SN-38 as well as 5-fluorouracil and oxaliplatin exhibit a good distribution

386 in the SR area in the plot (Figure 5C and D). However, the remaining values show a

387 worse distribution. In addition, their frequency distributions are distinct from an ideal 
388 distribution (Figure 5A and B). Nevertheless, comparing their correlation coefficients

389 and the accuracy of resistance classification to the diverse carboplatin combinations

390 reveals that both 5-fluorouracil combinations lie in a similar range (Table 3). The high

391 correlation coefficients are probably due to the good distribution of values in the SR

392 area. These results are in agreement with the results of the different carboplatin

393 combinations and support our previous assumptions.

394 In clinical practice drug combinations are often applied and therefore it is necessary to 395 have an approach for testing drug combinations to provide the best treatment for

396 individual patients. Regarding our results, produced by investigating different

397 combinations and tumor types, such an approach could be offered by our presented

398 system in a clinically relevant setting. In contrast to our system, other methods to test

399 drug combinations are far away from testing in a clinically relevant way since they use

400 cell lines instead of tumor samples (Edelman, Quam \& Mullins, 2001; Kashif et al., 2015;

401 Patra et al., 2016). However, our system still has to be validated by clinical data in order 402 to prove its efficacy for a patient.

403 The basis of our system is an additivity concept. In case of the different tested 404 combinations, which function in an additive way, it is sufficient to measure the single 405 drugs and calculate the PCls of the corresponding combinations as long as the single 406 drugs both are classified either as SR or ER. Thereby it is possible to find the best or the 407 worst combination, respectively. When single drugs are both classified as MR or belong 408 to distinct resistance categories it is reasonable to measure the combination. 409 Furthermore, if there is a priority for a specific combination as it is the case for colon 410 carcinoma, which is by default treated either with 5-fluorouracil and SN-38 or 5411 fluorouracil and oxaliplatin, the combination could be tested in the first place.

412 However, when tested combined drugs do not function in an additive way, this is 
413 resulting in possible limitations of our system and measurement of single drugs might

414 not be sufficient. This effect was seen when we tested the combinations carboplatin and

415 docetaxel as well as carboplatin and gemcitabine. These combinations exhibit frequency

416 distributions which differ in a great extent from an ideal distribution (Figure 4A and B).

417 For example, the mean $\mathrm{PCl}$ values for gemcitabine and the combination with carboplatin

418 are very high and the curves are shifted to the right (Figure 4B and E). The data points

419 in the plot are shifted to the right as well (Figure 4D). A distinct distribution in the plot is

420 seen for the data points of the combination carboplatin and docetaxel (Figure 4C). In

421 contrast to all other combinations, a data point from a single patient classified as SR for

422 both drugs lies in the measured ER range. Additionally, the correlation coefficients and

423 the accuracy of resistance classification are also worse than for the other tested

424 combinations (Table 2). These discrepancies could be explained by a non-additive

425 mode of action of the used combinations. In case of gemcitabine and carboplatin

426 synergism is reported for cell line systems (Edelman, Quam \& Mullins, 2001; de Brito

427 Galvao et al., 2012; Jin et al., 2013; Tomita et al., 2014). The reason for the false

428 classification in case of docetaxel could be an antagonistic effect, as shown before

429 (Budman, Calabro \& Kreis, 2002). Thus, calculating PCl values of a combination is not

430 applicable in a non-additive situation since this might lead to false results. Therefore, if it

431 is previously known that two drugs do not function in an additive way when applied in

432 combination, the combination needs to be tested directly. Thereby, via measuring the

433 combination it might be possible to predict the efficacy of the combination.

434 Our previous results and conclusions are perfectly supported by Bland-Altman-Plots

435 (Supplemental Figure 1). For the non-additive combinations carboplatin and

436 gemcitabine and carboplatin and docetaxel, which showed poor correlation coefficients

437 and accuracy of resistance classification, the plots revealed also significant differences 
438 between calculated and measured values. All other combinations that showed good

439 correlation coefficients and accuracy of resistance classification, performed well in the

440 plots and showed a good agreement between calculated and measured values. For the

441 combination carboplatin and docetaxel there is almost no difference between calculated

442 and measured values. The larger differences of the other additive combinations may be

443 due to the fact that our system is only functional when the single drugs are both

444 classified as ER or SR. In case of MR or divergent resistance categories, the 445 classification is less precise and leads to discrepancies. Therefore, the results of the

446 Bland-Altman-Plots suggest, that our new system, including the way of analysis, is

447 functional in predicting the efficacy of additive drug combinations when single drugs are 448 classified either as SR or ER.

449 Moreover, our new system could be employed to identify unknown non-additive 450 combinations by comparing measured and calculated values. A low correlation 451 coefficient, a low agreement between calculated and measured values as shown in a 452 Bland-Altman-Plot would indicate a variation from an additive situation. A method to 453 improve the identification of non-additive combinations would be to use only the 454 calculated values for determining the resistance borders and not consider the borders 455 determined by the measured values. In case of synergistic effects the calculated border 456 would classify more data points as SR than in the measured setting. With the other 457 borders extreme high $\mathrm{PCl}$ values could have been classified as extreme resistance even 458 though it is quite likely the combination is able to produce an effect. The same is the 459 case for antagonistic effects, more values would be classified as ER by the use of the 460 calculated borders. Therefore, antagonistic combinations would not be used for 461 treatment. These assumptions still have to be validated by clinical data.

462 Nevertheless a practical approach for measuring and evaluating the efficacy of any drug 
463 combination - even for unknown drug combinations - could be the following approach.

464 Since the resistance borders (SR/MR and MR/ER) of the single drugs are known, the 465 resistance borders of any drug combination can be computed by calculating the means 466 of the resistance borders of the single drugs, which are part of a certain combination. 467 That means that for the future only the resistance borders for single drugs need to be 468 measured and the resistance borders of drug combinations can be calculated. This 469 would be a tremendous reduction in complexity and effort, since the resistance borders 470 of any drug combination do not need to be measured any more.

471 In general, one can conclude that our system might be able on the one hand to measure 472 single drugs and calculate the efficacy of their combination in an additive situation if the 473 single drugs are both categorized as SR or ER. Thereby, the best and worst combination 474 can be found. These data are backed up with clinical data in which the best single drugs 475 were used for therapy in clinical trial settings (Orr, Orr \& Kern, 1999; Mehta et al., 2001; 476 Holloway et al., 2002; Loizzi et al., 2003; d'Amato et al., 2009; Kim et al., 2009; Matsuo 477 et al., 2009). On the other hand, it could be employed to measure drug combinations if 478 necessary, for example in case of other resistance categories or if a specific combination 479 is preferred. If feasible, the single drugs should be measured in addition to the 480 combination when it is planned to treat a patient with a certain combination therapy. 481 Thereby, it is possible to detect which of the single drugs is not functional in case of a 482 non-responding combination. Therefore, this system could be applied in order to detect 483 chemotherapy resistances of single patients and help to provide the best therapy option 484 for the individual patient. Despite the usefulness of single drug efficacy measurements, 485 in the future this system has to be further validated by clinical data to prove that it can 486 further improve clinical benefit.

487 Additionally, the use of this test could be extended to predict treatment outcomes of 
488 treatment plans with more than two drugs involved. Therefore, the used monotherapy's

489 concentration of the single drugs measured in combination, could be divided by the

490 number of used drugs in this multi-component treatment. The extension of this model

491 would help to close the gap between clinic and diagnostic even further.

492 However, it cannot be guaranteed that a patient responds to a chemotherapy when he is

493 sensitive to a specific chemotherapeutic or combination since this test system is used to

494 detect resistances rather then to identify presumably effective chemotherapeutics. If a

495 therapy is effective could for example be influenced by the mode of application of the

496 chemotherapeutics. When drugs are not effective in vitro, the patient will not respond to

497 the therapy, independent on the application mode. If a drug is effective in vitro but is not

498 applied effectively enough, the application mode could affect the efficacy of the therapy.

499 Moreover, an in vitro system is not able to mimic all resistance mechanisms in the body,

500 like the detoxification capability of a patient for example. Thus, to further improve the

501 individualized treatment of cancer patients one could combine our test system to detect

502 resistances against chemotherapeutics with pharmacological and/or toxicological

503 investigations.

504 Last but not least, this method is not limited to chemotherapeutic drugs, but can be used

505 for any anti-cancer drug combinations - including new targeted drugs - which act directly

506 on the tumor cells.

d'Amato TA., Pettiford BL., Schuchert MJ., Parker R., Ricketts WA., Luketich JD., 
Landreneau RJ. 2009. Survival among patients with platinum resistant, locally advanced non-small cell lung cancer treated with platinum-based systemic therapy. Annals of Surgical Oncology 16:2848-2855. DOI: 10.1245/s10434-0090608-0.

Beck WT. 1987. The cell biology of multiple drug resistance. Biochemical Pharmacology 36:2879-2887.

Berenbaum MC. 1989. What is synergy? Pharmacological Reviews 41:93-141.

Blom K., Nygren P., Alvarsson J., Larsson R., Andersson CR. 2016. Ex Vivo Assessment of Drug Activity in Patient Tumor Cells as a Basis for Tailored Cancer Therapy. Journal of Laboratory Automation 21:178-187. DOI: 10.1177/2211068215598117. du Bois A., Lück H-J., Meier W., Adams H-P., Möbus V., Costa S., Bauknecht T., Richter B., Warm M., Schröder W., Olbricht S., Nitz U., Jackisch C., Emons G., Wagner U., Kuhn W., Pfisterer J., Arbeitsgemeinschaft Gynäkologische Onkologie Ovarian Cancer Study Group 2003. A randomized clinical trial of cisplatin/paclitaxel versus carboplatin/paclitaxel as first-line treatment of ovarian cancer. Journal of the National Cancer Institute 95:1320-1329.

du Bois A., Weber B., Rochon J., Meier W., Goupil A., Olbricht S., Barats J-C., Kuhn W., Orfeuvre H., Wagner U., Richter B., Lueck H-J., Pfisterer J., Costa S., Schroeder W., Kimmig R., Pujade-Lauraine E., Arbeitsgemeinschaft Gynaekologische Onkologie, Ovarian Cancer Study Group, Groupe d'Investigateurs Nationaux pour l'Etude des Cancers Ovariens 2006. Addition of epirubicin as a third drug to carboplatin-paclitaxel in first-line treatment of advanced ovarian cancer: a prospectively randomized gynecologic cancer intergroup trial by the Arbeitsgemeinschaft Gynaekologische Onkologie Ovarian Cancer Study Group and the Groupe d'Investigateurs Nationaux pour l'Etude des Cancers Ovariens. Journal of Clinical Oncology: Official Journal of the American Society of Clinical Oncology 24:1127-1135. DOI: 10.1200/JCO.2005.03.2938.

Bookman MA., Brady MF., McGuire WP., Harper PG., Alberts DS., Friedlander M., 
Colombo N., Fowler JM., Argenta PA., De Geest K., Mutch DG., Burger RA., Swart AM., Trimble EL., Accario-Winslow C., Roth LM. 2009. Evaluation of new platinum-based treatment regimens in advanced-stage ovarian cancer: a Phase III Trial of the Gynecologic Cancer Intergroup. Journal of Clinical Oncology: Official Journal of the American Society of Clinical Oncology 27:1419-1425. DOI: 10.1200/JCO.2008.19.1684.

de Brito Galvao JF., Kisseberth WC., Murahari S., Sutayatram S., Chew DJ., Inpanbutr N. 2012. Effects of gemcitabine and gemcitabine in combination with carboplatin on five canine transitional cell carcinoma cell lines. American Journal of Veterinary Research 73:1262-1272. DOI: 10.2460/ajvr.73.8.1262.

Budman DR., Calabro A., Kreis W. 2002. Synergistic and antagonistic combinations of drugs in human prostate cancer cell lines in vitro. Anti-Cancer Drugs 13:10111016.

Chou T-C. 2010. Drug combination studies and their synergy quantification using the Chou-Talalay method. Cancer Research 70:440-446. DOI: 10.1158/00085472.CAN-09-1947.

Edelman MJ., Quam H., Mullins B. 2001. Interactions of gemcitabine, carboplatin and paclitaxel in molecularly defined non-small-cell lung cancer cell lines. Cancer Chemotherapy and Pharmacology 48:141-144.

Grendys EC., Fiorica JV., Orr JW., Holloway R., Wang D., Tian C., Chan JK., Herzog TJ. 2014. Overview of a chemoresponse assay in ovarian cancer. Clinical \& Translational Oncology: Official Publication of the Federation of Spanish Oncology Societies and of the National Cancer Institute of Mexico 16:761-769. DOI: 10.1007/s12094-014-1192-8.

Holloway RW., Mehta RS., Finkler NJ., Li K-T., McLaren CE., Parker RJ., Fruehauf JP. 2002. Association between in vitro platinum resistance in the EDR assay and clinical outcomes for ovarian cancer patients. Gynecologic Oncology 87:8-16.

loannidis JPA. 2007. Is molecular profiling ready for use in clinical decision making? The 
Oncologist 12:301-311. DOI: 10.1634/theoncologist.12-3-301.

Jin G., Zhao J., Qi H., Lou M., Liu X., Qu Y., Zhao L., Zhang W., Shao J., Zhong H. 2013. Gemcitabine and carboplatin demonstrate synergistic cytotoxicity in cervical cancer cells by inhibiting DNA synthesis and increasing cell apoptosis. OncoTargets and Therapy 6:1707-1717. DOI: 10.2147/OTT.S54217.

Kashif M., Andersson C., Åberg M., Nygren P., Sjöblom T., Hammerling U., Larsson R., Gustafsson MG. 2014. A pragmatic definition of therapeutic synergy suitable for clinically relevant in vitro multicompound analyses. Molecular Cancer Therapeutics 13:1964-1976. DOI: 10.1158/1535-7163.MCT-13-0430.

Kashif M., Andersson C., Hassan S., Karlsson H., Senkowski W., Fryknäs M., Nygren P., Larsson R., Gustafsson MG. 2015. In vitro discovery of promising anti-cancer drug combinations using iterative maximisation of a therapeutic index. Scientific Reports 5:14118. DOI: 10.1038/srep14118.

Kern DH., Weisenthal LM. 1990. Highly specific prediction of antineoplastic drug resistance with an in vitro assay using suprapharmacologic drug exposures. Journal of the National Cancer Institute 82:582-588.

Kim HS., Kim TJ., Chung HH., Kim JW., Kim BG., Park NH., Song YS., Bae DS., Kang SB. 2009. In vitro extreme drug resistance assay to taxanes or platinum compounds for the prediction of clinical outcomes in epithelial ovarian cancer: a prospective cohort study. Journal of Cancer Research and Clinical Oncology 135:1513-1520. DOI: 10.1007/s00432-009-0598-0.

Loewe S. 1953. The problem of synergism and antagonism of combined drugs. Arzneimittel-Forschung 3:285-290.

Loizzi V., Chan JK., Osann K., Cappuccini F., DiSaia PJ., Berman ML. 2003. Survival outcomes in patients with recurrent ovarian cancer who were treated with chemoresistance assay-guided chemotherapy. American Journal of Obstetrics and Gynecology 189:1301-1307.

Ludwig JA., Weinstein JN. 2005. Biomarkers in cancer staging, prognosis and treatment 
selection. Nature Reviews. Cancer 5:845-856. DOI: 10.1038/nrc1739.

Matsuo K., Bond VK., Eno ML., Im DD., Rosenshein NB. 2009. Low drug resistance to both platinum and taxane chemotherapy on an in vitro drug resistance assay predicts improved survival in patients with advanced epithelial ovarian, fallopian and peritoneal cancer. International Journal of Cancer 125:2721-2727. DOI: 10.1002/ijc.24654.

Mehta RS., Bornstein R., Yu IR., Parker RJ., McLaren CE., Nguyen KP., Li KT., Fruehauf JP. 2001. Breast cancer survival and in vitro tumor response in the extreme drug resistance assay. Breast Cancer Research and Treatment 66:225-237.

Myers C., Cowan K., Sinha B., Chabner B. 1987. The phenomenon of pleiotropic drug resistance. Important Advances in Oncology:27-38.

Nygren P., Fridborg H., Csoka K., Sundström C., de la Torre M., Kristensen J., Bergh J., Hagberg H., Glimelius B., Rastad J. 1994. Detection of tumor-specific cytotoxic drug activity in vitro using the fluorometric microculture cytotoxicity assay and primary cultures of tumor cells from patients. International Journal of Cancer 56:715-720.

Nygren P., Larsson R. 2008. Predictive tests for individualization of pharmacological cancer treatment. Expert Opinion on Medical Diagnostics 2:349-360. DOI: 10.1517/17530059.2.4.349.

Orr JW., Orr P., Kern DH. 1999. Cost-effective treatment of women with advanced ovarian cancer by cytoreductive surgery and chemotherapy directed by an in vitro assay for drug resistance. The Cancer Journal from Scientific American 5:174178.

Patra B., Peng C-C., Liao W-H., Lee C-H., Tung Y-C. 2016. Drug testing and flow cytometry analysis on a large number of uniform sized tumor spheroids using a microfluidic device. Scientific Reports 6:21061. DOI: 10.1038/srep21061.

Pfisterer J., Weber B., Reuss A., Kimmig R., du Bois A., Wagner U., Bourgeois H., Meier W., Costa S., Blohmer J-U., Lortholary A., Olbricht S., Stähle A., Jackisch C., 
Hardy-Bessard A-C., Möbus V., Quaas J., Richter B., Schröder W., Geay J-F., Lück H-J., Kuhn W., Meden H., Nitz U., Pujade-Lauraine E., AGO-OVAR, GINECO 2006. Randomized phase III trial of topotecan following carboplatin and paclitaxel in first-line treatment of advanced ovarian cancer: a gynecologic cancer intergroup trial of the AGO-OVAR and GINECO. Journal of the National Cancer Institute 98:1036-1045. DOI: 10.1093/jnci/djj296.

Prisant LM. 2002. Fixed low-dose combination in first-line treatment of hypertension. Journal of Hypertension. Supplement: Official Journal of the International Society of Hypertension 20:S11-19.

Pusztai L., Rouzier R., Wagner P., Symmans WF. 2004. Individualized chemotherapy treatment for breast cancer: is it necessary? Is it feasible? Drug Resistance Updates: Reviews and Commentaries in Antimicrobial and Anticancer Chemotherapy 7:325-331. DOI: 10.1016/j.drup.2004.10.002.

Rutherford T., Orr J., Grendys E., Edwards R., Krivak TC., Holloway R., Moore RG., Puls L., Tillmanns T., Schink JC., Brower SL., Tian C., Herzog TJ. 2013. A prospective study evaluating the clinical relevance of a chemoresponse assay for treatment of patients with persistent or recurrent ovarian cancer. Gynecologic Oncology 131:362-367. DOI: 10.1016/j.ygyno.2013.08.009.

Sparano JA. 1999. Doxorubicin/taxane combinations: cardiac toxicity and pharmacokinetics. Seminars in Oncology 26:14-19.

Tallarida RJ. 2006. An overview of drug combination analysis with isobolograms. The Journal of Pharmacology and Experimental Therapeutics 319:1-7. DOI: 10.1124/jpet.106.104117.

Tattersall MH., Harnett PR. 1986. Drug resistance in the clinical situation. Progress in Clinical and Biological Research 223:151-159.

Tomita Y., Saito T., Okadome M., Eto T., Ariyoshi K., Shimamoto K. 2014. The safety and efficacy of cisplatin plus gemcitabine in recurrent ovarian cancer. International Journal of Clinical Oncology 19:662-666. DOI: 10.1007/s10147-013-0599-5. 
Trusheim MR., Berndt ER., Douglas FL. 2007. Stratified medicine: strategic and economic implications of combining drugs and clinical biomarkers. Nature Reviews. Drug Discovery 6:287-293. DOI: 10.1038/nrd2251. 


\section{Figure 1 (on next page)}

New system for testing drug combinations in vitro with the CTR-Test.

(A) Ideal distribution of percent cell growth inhibition ( $\mathrm{PCl}$ ) values from a tumor patient collective using the ideal concentration of a drug (black curve, mean at $50 \% \mathrm{PCl}$ ). Two individually measured drugs $A$ and $B$ at ideal concentrations show identical ideal distribution curves (black curve). One half of the ideal concentration of drug A can be replaced by one half of the ideal concentration of drug B or vice versa when the drugs are applied in combination. This results in a curve identical to single curves of A or B (black curve, additive effect). A curve shift to the left would be due to an antagonistic effect, a shift to the right would be due to a synergistic effect when two drugs are combined (grey dashed curves). In an ideal situation the measured $\mathrm{PCl}$ values are equal to the calculated $\mathrm{PCl}$ values (formula see text) of a drug combination. The ideal PCl values plotted against each other result in a correlation coefficient of 1 (additive effect). Data points above or below the line show an antagonistic or synergistic effect, respectively. (C) 273 ovarian carcinoma samples (99 primary, 140 recurrent and 34 unknown ovarian carcinoma cases) were treated with carboplatin and paclitaxel alone or in combination. $\mathrm{PCl}$ values of the single drugs were determined with the CTR-test. The PCI values of the combination were measured with the CTR-test as well as calculated via the presented formula. For measuring the combination, half of the concentration of each drug was used. The frequency distributions of $\mathrm{PCl}$ values of the different settings were plotted (black dashed curve: carboplatin alone, grey dashed curve: paclitaxel alone, grey curve: measured PCl of combination, black curve: calculated PCI of combination). The mean $\mathrm{PCl}$ value of all curves spreads around $70 \%$. (D) The measured and calculated $\mathrm{PCl}$ values of the combination were plotted against each other resulting in a correlation coefficient of 0.84 (Table 1). 

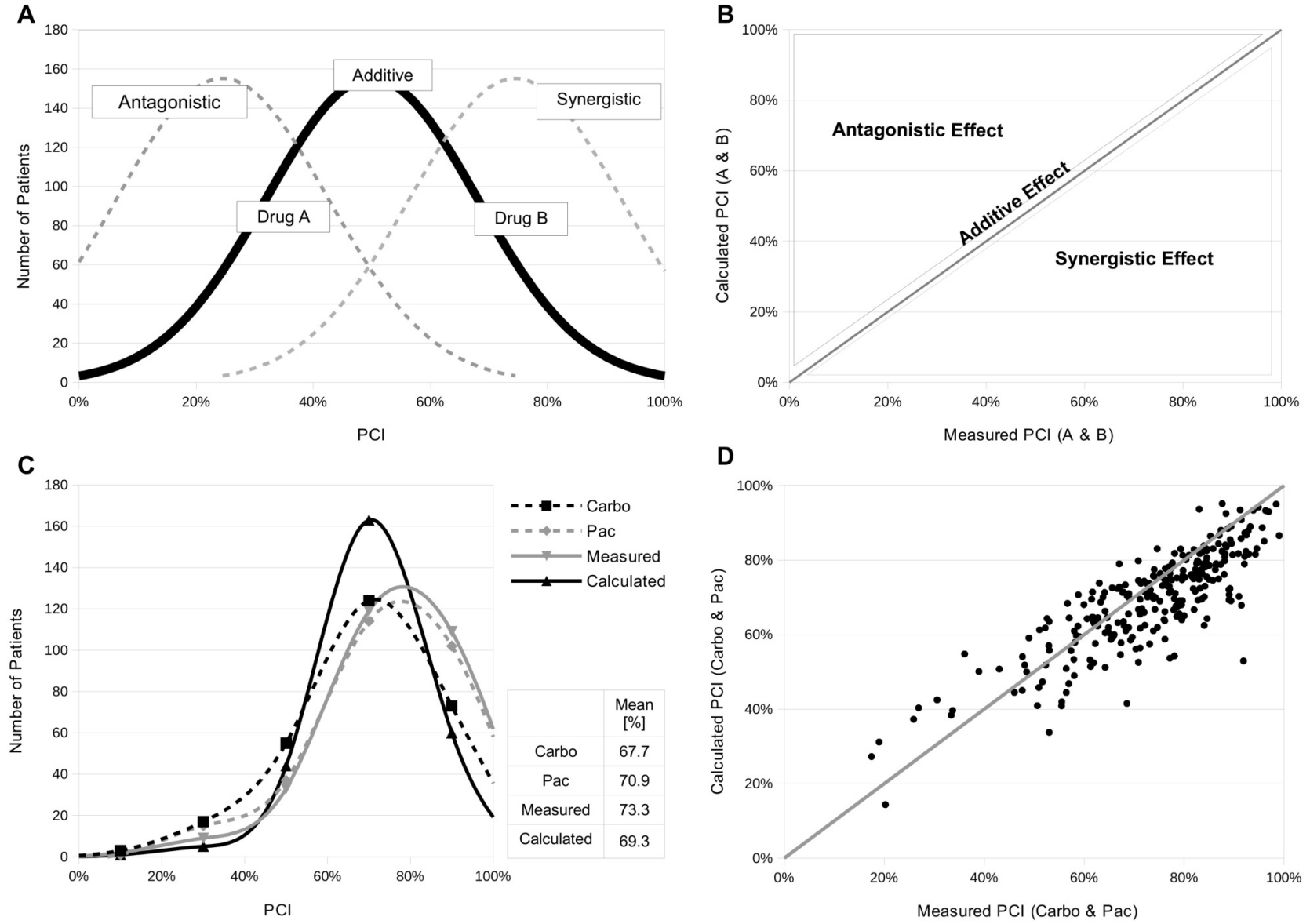


\section{Figure 2}

Evaluation of predicting efficacy of the new system for chemoresistance classification.

(A) The distribution of $\mathrm{PCl}$ values of a tumor patient collective for a certain drug applied at a specific concentration is used to classify three chemoresistance categories. ER (extreme resistance) is characterized as $\mathrm{PCl}<\mu$ (mean) - $1 \mathrm{SD}$ (standard deviation) (dashed red line). $\mathrm{PCl}>\mathrm{ER}$ but $<\mu$ (dashed green line) is classified as MR (medium resistance), $\mathrm{PCl} \geq \mu$ is classified as SR (slight resistance). (B and C) Data set contains 273 ovarian carcinoma samples (B) The resistance categories of this data set for carboplatin alone, paclitaxel alone, measured and calculated combination were defined by the system described in (A) and the classification borders for measured and calculated are marked by a green $(\mu)$ and red line $(\mu$ - 1 SD). The underlying curves are presented in Figure $1 \mathrm{C}$. These measured and calculated $\mathrm{PCl}$ values of the combination carboplatin and paclitaxel are plotted against each other. Individual data points are color-coded depending on the chemoresistance category of the patient for the two single drugs. (C) Single drug resistance categories are compared to the measured categories of the combination carboplatin and paclitaxel. 

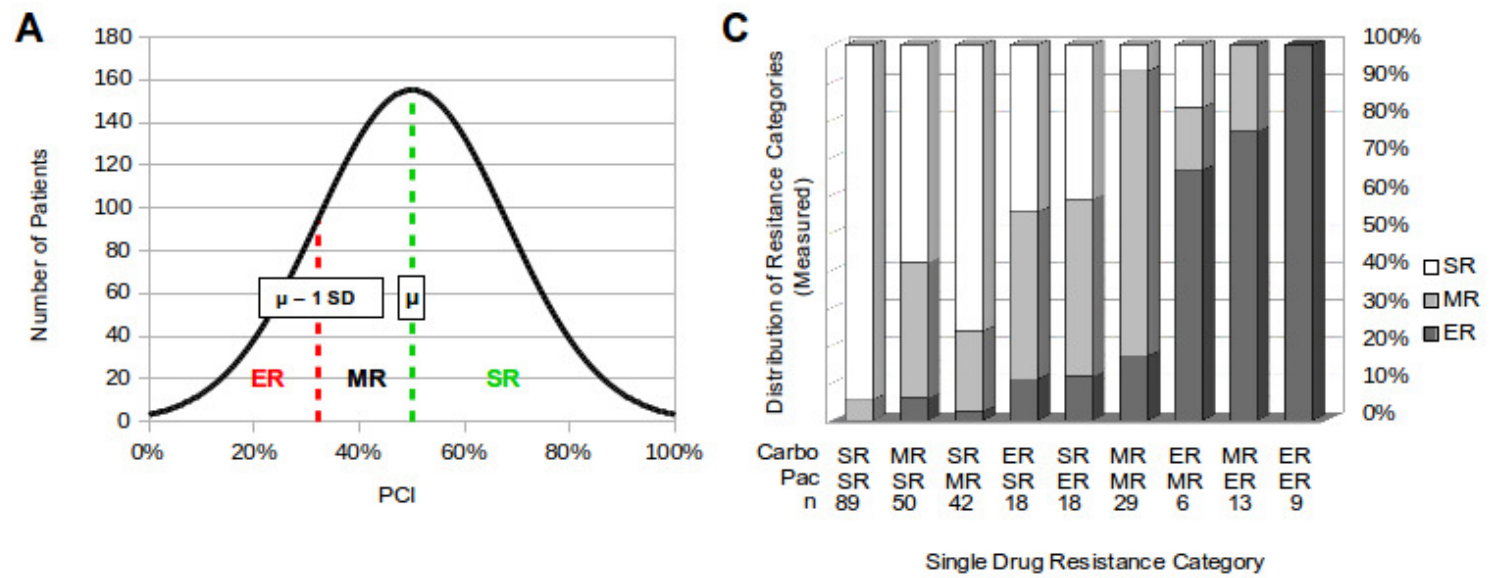

B

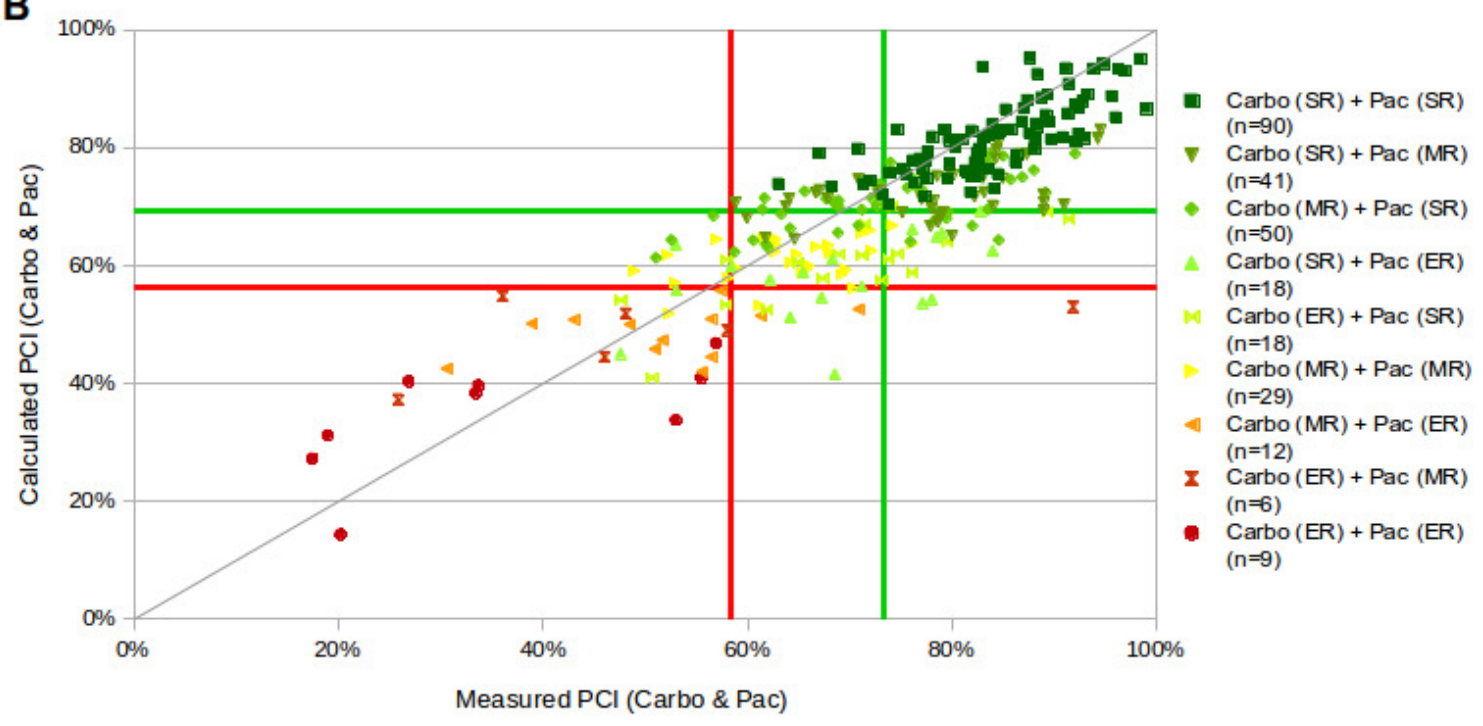




\section{Figure 3 (on next page)}

New system is used to test other drug combinations.

(A - D) 39 ovarian carcinoma samples were treated with carboplatin and Caelyx (A), 29 with carboplatin and doxorubicin (B), 32 with carboplatin and etoposide (C) and 29 with carboplatin and topotecan (D) alone or in combination. $\mathrm{PCl}$ of the single drugs and the different combinations was measured with the CTR-Test. In addition, the PCI of the combinations was calculated with the presented formula (1). The frequency distributions of $\mathrm{PCl}$ values of the different settings were plotted (black dashed curves: carboplatin alone, grey dashed curves: diverse drugs alone, grey curves: measured PCl of combinations, black curves: calculated $\mathrm{PCl}$ of combinations). The mean $\mathrm{PCl}$ values for all curves can be seen in I. (E - H) These distribution curves of $\mathrm{PCl}$ values for the different drugs alone, measured and calculated combinations with carboplatin were used to define resistance categories via the system described in Figure 2A. The classification borders for measured and calculated are marked by a green $(\mu)$ and red line ( $\mu-1$ SD). The measured and calculated PCI values of the combinations carboplatin and one of the other four drugs are plotted against each other. Individual data points are color-coded depending on the chemoresistance category of the patient for the two single drugs. (I) Table representing the mean $\mathrm{PCl}$ values for all curves. 

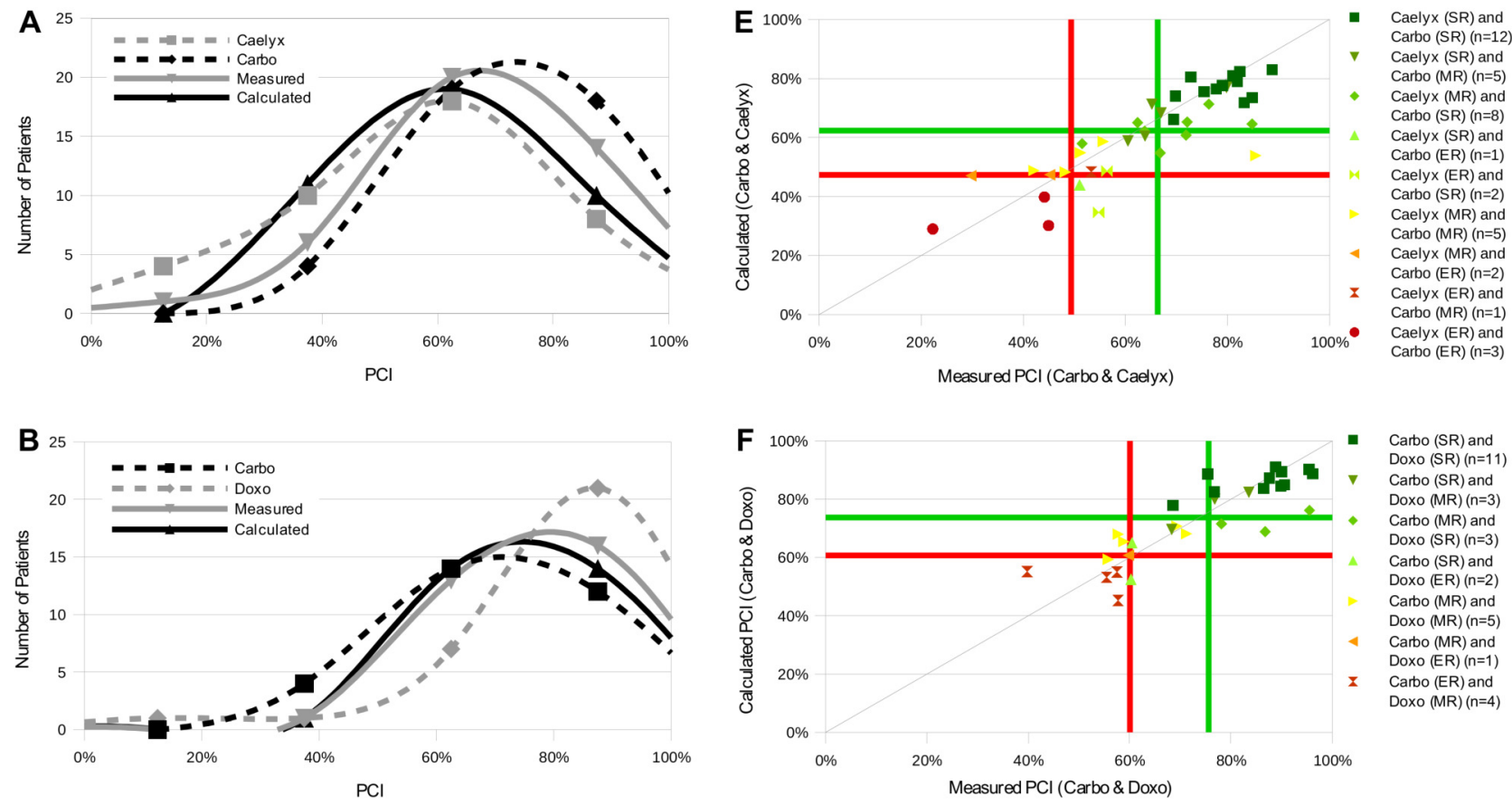

Carbo (SR) and Doxo (SR) (n=11 Carbo (SR) and Doxo (MR) (n=3) Carbo (MR) and Doxo (SR) $(n=3)$ Carbo (SR) and Doxo (ER) $(n=2)$ Carbo (MR) and Doxo (MR) (n=5) Carbo (MR) and Doxo (ER) $(n=1)$

Carbo (ER) and

Doxo (MR) (n=4)
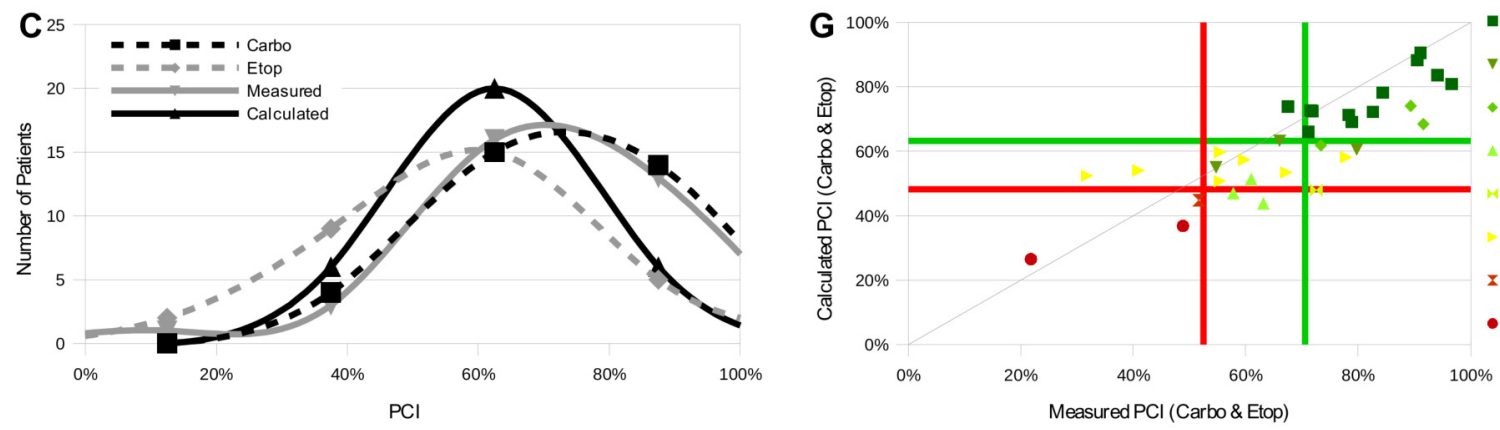

Carbo (SR) and top (SR) (n=12) Carbo (SR) and Etop (MR) $(\mathrm{n}=3)$ Carbo (MR) and Etop (SR) $(\mathrm{n}=3)$ Carbo (SR) and Etop (ER) $(n=3)$ Carbo (ER) and Etop (SR) (n=1) Carbo (MR) an Etop (MR) $(n=7)$ Carbo (ER) and Etop (MR) $(n=1)$ Carbo (ER) and Etop $(E R)(n=2)$
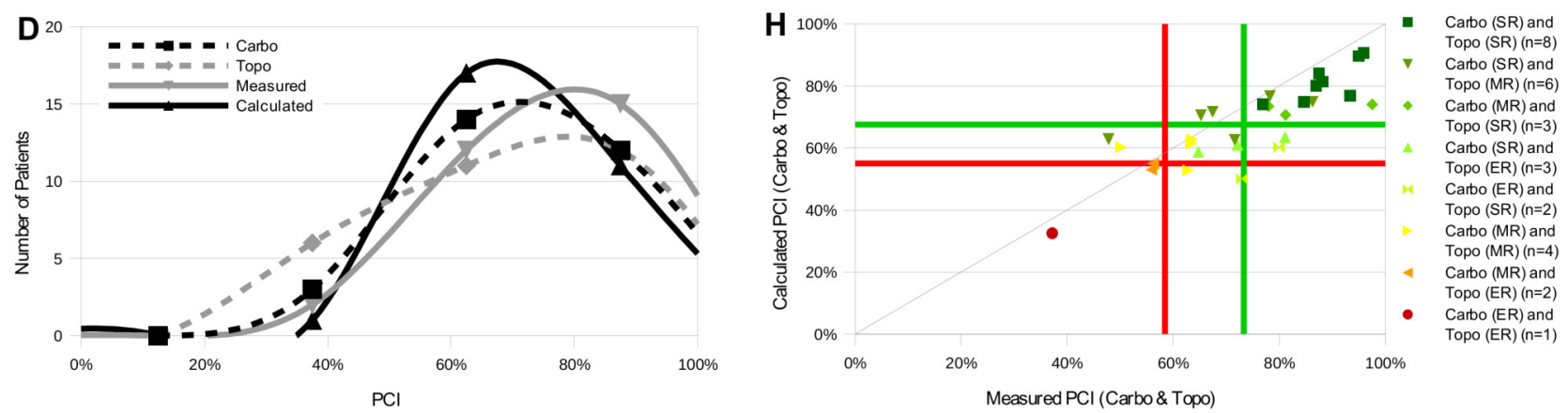

I

\begin{tabular}{|c|c|c|c|c|}
\hline & $\begin{array}{c}\text { Carbo \& } \\
\text { Caelyx }\end{array}$ & $\begin{array}{c}\text { Carbo \& } \\
\text { Doxo }\end{array}$ & $\begin{array}{c}\text { Carbo \& } \\
\text { Etop }\end{array}$ & $\begin{array}{c}\text { Carbo \& } \\
\text { Topo }\end{array}$ \\
\hline $\begin{array}{c}\text { Carbo } \\
\text { (Mean [\%]) }\end{array}$ & 68.1 & 67.0 & 67.6 & 67.3 \\
\hline $\begin{array}{c}\text { Second Drug } \\
\text { (Mean [\%]) }\end{array}$ & 54.6 & 77.7 & 57.3 & 67.9 \\
\hline $\begin{array}{c}\text { Measured } \\
\text { (Mean [\%]) }\end{array}$ & 64.6 & 74.2 & 69.5 & 73.9 \\
\hline $\begin{array}{c}\text { Calculated } \\
\text { (Mean [\%]) }\end{array}$ & 61.3 & 72.9 & 62.1 & 67.6 \\
\hline
\end{tabular}




\section{Figure 4(on next page)}

New system is used to test other drug combinations (exceptions).

(A and B) 30 ovarian carcinoma samples were treated with carboplatin and docetaxel (A), 36 with carboplatin and gemcitabine (B) alone or in combination. $\mathrm{PCl}$ of the single drugs and the two combinations was measured with the CTR-Test. Additionally, the PCl of the combinations was calculated with the presented formula. The frequency distributions of $\mathrm{PCl}$ values of the different settings were plotted (black dashed curves: carboplatin alone, grey dashed curves: docetaxel or gemcitabine alone, grey curves: measured PCl of combinations, black curves: calculated $\mathrm{PCl}$ of combinations). The mean $\mathrm{PCl}$ values for all curves can be seen in $\mathrm{E}$. (C and D) The distribution curves of $\mathrm{PCl}$ values for the two drugs alone, measured and calculated combinations with carboplatin, were used to define resistance categories via the system described in Figure 2A. The classification borders for measured and calculated are marked by a green $(\mu)$ and red line ( $\mu-1$ SD). The measured and calculated PCI values of the combinations carboplatin and one of the two other drugs are plotted against each other. Individual data points are color-coded depending on the chemoresistance category of the patient for the single drugs. (E) Table representing the mean $\mathrm{PCl}$ values for all curves. 

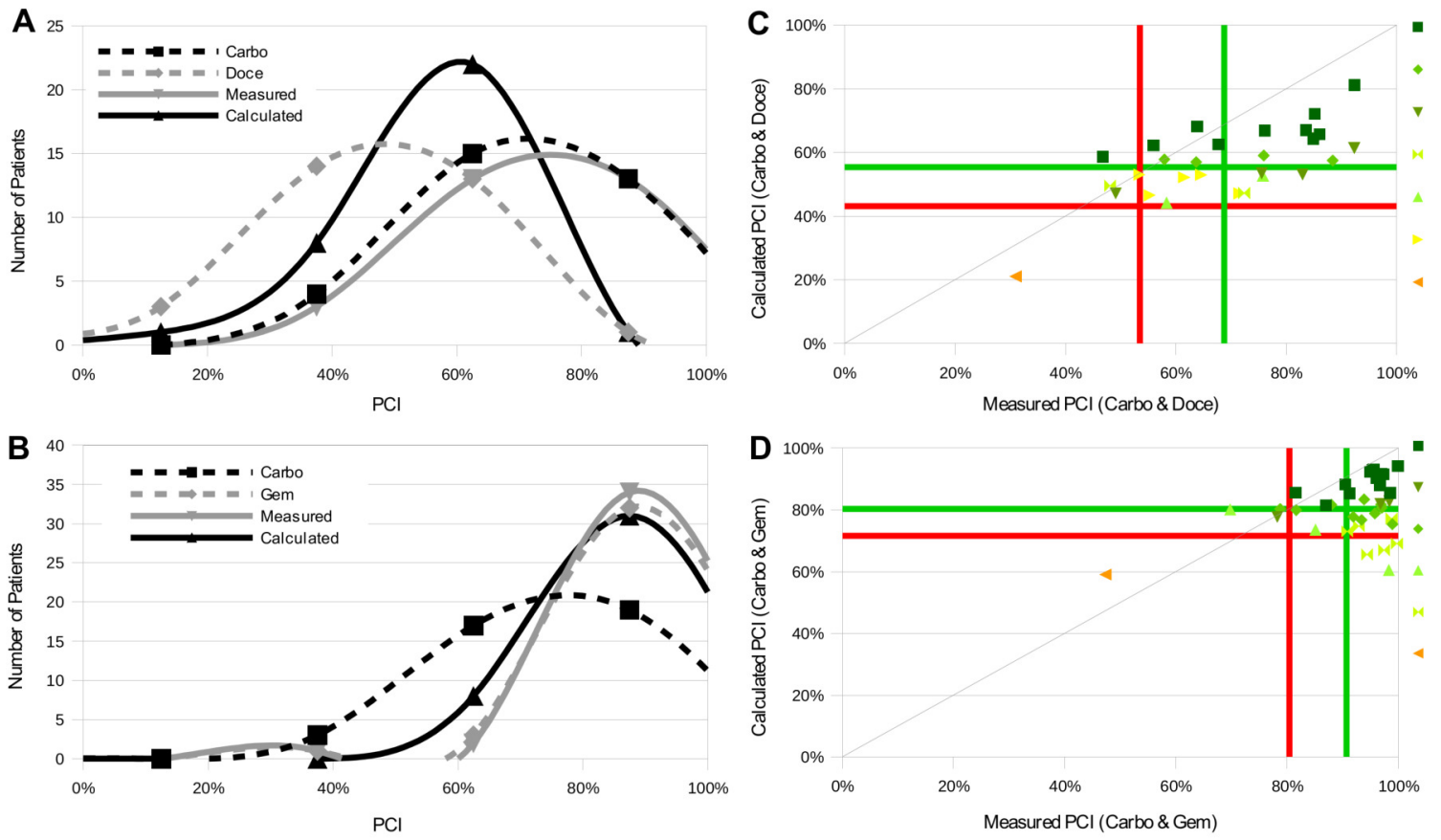

$\mathbf{E}$

\begin{tabular}{|r|c|c|}
\hline & Carbo \& Doce & Carbo \& Gem \\
\hline Carbo (Mean [\%]) & 67.3 & 69.6 \\
\hline Second Drug (Mean [\%]) & 42.2 & 91.4 \\
\hline Measured (Mean [\%]) & 69.2 & 91.2 \\
\hline Calculated (Mean [\%]) & 54.8 & 80.5 \\
\hline
\end{tabular}




\section{Figure $\mathbf{5}$ (on next page)}

New system is used to test other carboplatin-independent drug combinations.

(A and B) 32 ovarian carcinoma, 1 melanoma, 1 small cell bronchial carcinoma, 1, non-small cell lung carcinoma, 1 mamma carcinoma and 4 colon carcinoma were treated with 5fluorouracil and SN-38 (A); 31 ovarian carcinoma, 1 melanoma, 1 small cell bronchial carcinoma, 1 non-small cell lung carcinoma, 1 mamma carcinoma and 2 colon carcinoma were treated with 5-fluorouracil and oxaliplatin (B) alone or in combination. $\mathrm{PCl}$ of the single drugs and the two combinations was measured with the CTR-Test and the PCl of the two combinations was also calculated with the presented formula. The frequency distributions of $\mathrm{PCl}$ values of the different settings were plotted (black dashed curves: 5-fluorouracil alone, grey dashed curves: SN-38 or oxaliplatin alone, grey curves: measured PCl of combinations, black curves: calculated $\mathrm{PCl}$ of combinations). The mean $\mathrm{PCl}$ values for all curves can be seen in $\mathrm{E}$. (C and $\mathbf{D}$ ) The distribution curves of $\mathrm{PCl}$ values for the two drugs alone, measured and calculated combinations with 5-fluorouracil, were used to define resistance categories via the system described in Figure 2A. The classification borders for measured and calculated are marked by a green $(\mu)$ and red line ( $\mu-1$ SD). The measured and calculated PCI values of the combinations 5-fluorouracil and one of the two other drugs are plotted against each other. Individual data points are color-coded depending on the chemoresistance category of the patient for the single drugs. (E) Table representing the mean $\mathrm{PCl}$ values for all curves. 

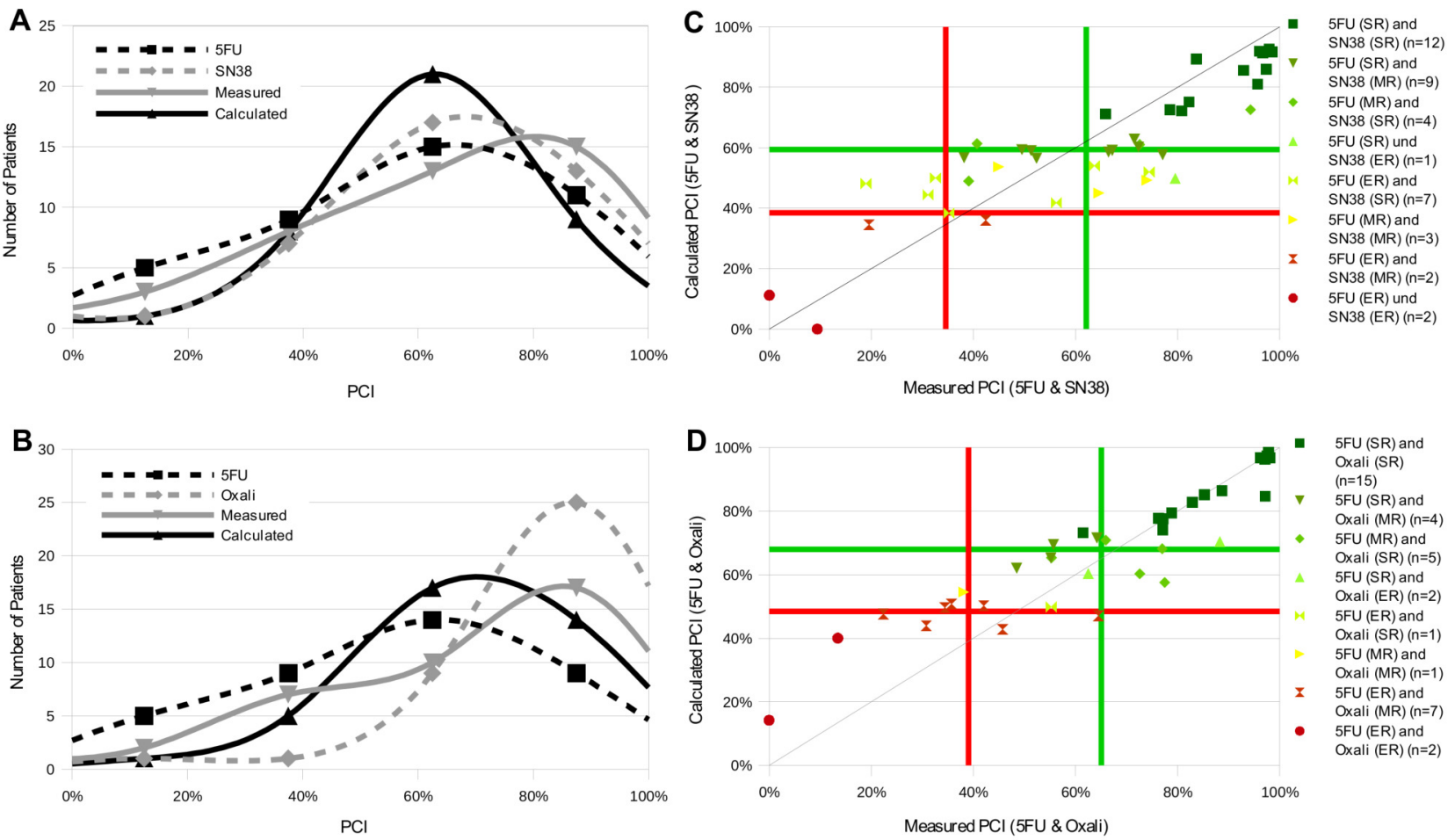

E

\begin{tabular}{|r|c|c|}
\hline & 5FU \& SN38 & 5FU \& Oxali \\
\hline 5FU (Mean [\%]) & 58.7 & 57.6 \\
\hline Second Drug (Mean [\%]) & 60.1 & 78.4 \\
\hline Measured (Mean [\%]) & 62.1 & 65.1 \\
\hline Calculated (Mean [\%]) & 59.4 & 68.0 \\
\hline
\end{tabular}


Table $\mathbf{1}$ (on next page)

Used chemotherapeutics and their concentration. 


\begin{tabular}{|c|c|c|c|}
\hline Name & Chemical class/mechanism of action & $\begin{array}{l}\text { Used Monotherapy } \\
\text { Concentration }[\mu \mathrm{g} / \mathrm{ml}]\end{array}$ & $\begin{array}{l}\text { Used combination } \\
\text { concentration }[\mu \mathrm{g} / \mathrm{ml}]\end{array}$ \\
\hline 5-Fluorouracil & Thymidylate synthase inhibitor/antimetabolites & 3.0 & 1.5 \\
\hline Carboplatin & $\begin{array}{l}\text { Platinum-based antineoplastic agent/ DNA } \\
\text { interaction and interference with DNA repair }\end{array}$ & 3.81 & 1.905 \\
\hline $\begin{array}{c}\text { Caelyx } ® \\
\text { (Doxorubicin - liposomal) }\end{array}$ & $\begin{array}{l}\text { Intercalating DNA/anthracycline antitumor } \\
\text { antibiotic }\end{array}$ & 3.62 & 1.81 \\
\hline Docetaxel & Interference in cell division & 1.94 & 0.97 \\
\hline Doxorubicin & $\begin{array}{l}\text { Intercalating DNA/anthracycline antitumor } \\
\text { antibiotic }\end{array}$ & 0.1 & 0.05 \\
\hline Etoposide & Topoisomerase inhibitor & 3.62 & 1.81 \\
\hline Gemcitabine & Nucleoside analog & 0.014 & 0.007 \\
\hline Oxaliplatin & $\begin{array}{l}\text { Platinum-based antineoplastic agent/ DNA } \\
\text { interaction and interference with DNA repair }\end{array}$ & 1 & 0.5 \\
\hline Paclitaxel & Interference in cell division & 2.1 & 1.05 \\
\hline Topotecan & Topoisomerase inhibitor & 0.1 & 0.05 \\
\hline SN-38 & Antineoplastic drug/ inhibition of topoisomerase 1 & 0.012 & 0.006 \\
\hline
\end{tabular}




\section{Table 2 (on next page)}

Correlation coefficient ( $R$ ) and resistance classification (calculated equal to measured values) of carboplatin combinations. 


\begin{tabular}{|c|c|c|c|c|c|c|c|}
\hline & $\begin{array}{c}\text { Carboplatin } \\
\text { \& Paclitaxel }\end{array}$ & $\begin{array}{c}\text { Carboplatin } \\
\text { \& Caelyx® }\end{array}$ & $\begin{array}{c}\text { Carboplatin } \\
\text { \& Doxorubicin }\end{array}$ & $\begin{array}{c}\text { Carboplatin } \\
\text { \& Etoposide }\end{array}$ & $\begin{array}{c}\text { Carboplatin } \\
\text { \& Topotecan }\end{array}$ & $\begin{array}{c}\text { Carboplatin } \\
\text { \& Docetaxel }\end{array}$ & $\begin{array}{c}\text { Carboplatin } \\
\text { \& Gemcitabine }\end{array}$ \\
$\begin{array}{c}\text { Correlation } \\
\text { coefficient (R) }\end{array}$ & 0.84 & 0.84 & 0.85 & 0.83 & 0.82 & 0.68 \\
$\begin{array}{c}\text { Resistance } \\
\text { classification: } \\
\text { calculated } \\
\text { measured }\end{array}$ & $77.0 \%$ & $72.5 \%$ & $71.0 \%$ & $68.8 \%$ & $74.2 \%$ & $64.3 \%$ \\
\hline $\begin{array}{c}\text { Data set size (n) } \\
\text { (n) }\end{array}$ & 273 & 39 & 29 & 32 & 29 & $48.7 \%$ \\
\hline
\end{tabular}




\section{Table 3(on next page)}

Correlation coefficient ( $R$ ) and resistance classification (calculated equal to measured values) of 5-fluorouracil combinations. 
Resistance classification: calculated $=$ measured

Data set size (n)

$6.7 \%$

$70.3 \%$ 